\title{
Gradhiva
}

GRADHIV

Revue d'anthropologie et d'histoire des arts

$24 \mid 2016$

ARTchives

\section{Arnaud Esquerre, Théorie des événements extraterrestres. Essai sur le récit fantastique}

Paris, Fayard, 2016

\section{Anne de Sales}

\section{(2) OpenEdition}

\section{Journals}

Édition électronique

URL : http://journals.openedition.org/gradhiva/3317

DOI : 10.4000/gradhiva.3317

ISSN : 1760-849X

\section{Éditeur}

Musée du quai Branly Jacques Chirac

Édition imprimée

Date de publication : 7 décembre 2016

Pagination : 258-259

ISBN : 978-2-35744-094-4

ISSN : 0764-8928

\section{Référence électronique}

Anne de Sales, "Arnaud Esquerre, Théorie des événements extraterrestres. Essai sur le récit fantastique », Gradhiva [En ligne], 24 | 2016, mis en ligne le 07 décembre 2016, consulté le 23 septembre 2020. URL : http://journals.openedition.org/gradhiva/3317 ; DOI : https://doi.org/10.4000/gradhiva.3317

Ce document a été généré automatiquement le 23 septembre 2020.

(C) musée du quai Branly 


\section{Arnaud Esquerre, Théorie des événements extraterrestres. Essai sur le récit fantastique}

Paris, Fayard, 2016

Anne de Sales

\section{RÉFÉRENCE}

Arnaud Esquerre, Théorie des événements extraterrestres. Essai sur le récit fantastique. Paris, Fayard, 2016, 260 p. 


\section{Arnaud Esquerre}

\section{Théorie des événements extraterrestres \\ Essai sur le récit fantastique}

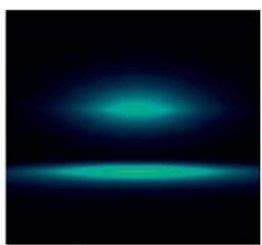

hdo

fayard

Plus de 1500 récits de témoins d'événements extraterrestres couvrant une période de 60 ans (1952-2012) sont archivés par le Groupe d'études et d'informations sur les phénomènes aérospatiaux non identifiés (Geipan), qui dépend du Centre national d'études spatiales (Cnes). Sur la base de ce corpus entré dans le domaine public, Arnaud Esquerre élabore une théorie des événements extraterrestres qu'il expose en deux parties. Dans la première, il s'attache à montrer que ces histoires, où les descriptions des choses énigmatiques sont très variées, constituent cependant une même classe de récits reposant sur un schéma narratif commun. Dans la seconde, il se penche sur le type d'enquêtes (militaire, scientifique, policière) appelées à éclaircir le mystère.

L'auteur s'inspire en premier lieu des travaux du linguiste William Labov (1967 et 2013) établissant que dans les récits oraux d'expériences vécues, l'ordre des événements dans le texte suit leur ordre chronologique : il n'y a donc ni anticipation ni flash-back. À ce premier trait structural fondamental s'ajoute la règle qui veut que ce ne soit pas l' apparition de la chose étrange dans le ciel qui capte l'attention mais au contraire sa disparition, ou ce que l'auteur appelle l'imperception. Cette soudaine soustraction à la vue laisse le témoin dans l'impossibilité d'identifier le phénomène et caractérise les récits directs d'événements extraterrestres. À cela s'ajoute que les actions des témoins sont insignifiantes, la vision est le seul sens quasiment sollicité, les répétitions sont nombreuses et enfin tous les détails mentionnés le sont en relation directe avec l'événement qu'il s'agit d'élucider. L'auteur qualifie ces détails d'«indiciaires » par opposition à ceux des récits littéraires consistant, par exemple, à produire des effets de réalité. Ainsi, qu'il s'agisse d'un récit oral retranscrit par les gendarmes sous forme de procès-verbal ou d'un questionnaire écrit auquel les témoins répondent librement depuis chez eux, le modèle narratif identifié reste le même. Celui-ci ne dépend donc pas des conditions d'énonciation, confirmant par là sa nature structurale; il ne s'est pas non plus modifié au cours du temps ou du moins pas depuis que ces récits sont archivés, à savoir la fin de la Seconde Guerre mondiale. Une autre caractéristique de ces 
récits est, pour finir, la dénégation par laquelle ils se terminent : invariablement, le narrateur (sans distinction de genre) insiste pour conclure qu'il ne croit pas aux extraterrestres, qu'il n'était pas ivre lors des faits ou qu'il n'est pas fou, c'est-à-dire qu'il a bien vu ce qu'il a vu, même s'il ne sait pas l'identifier. C'est en « rabat[tant] le récit du côté officiel du territoire de la vérité... » (p. 71) - les extraterrestres n'existent pas - que ce principe de dénégation (je ne crois pas aux extraterrestres, je n'ai pas été trompé par mes sens) introduit l'hypothèse de l'existence d'une vie extraterrestre (mais alors qu'était cet engin dans le ciel ?). Le narrateur, lui-même dans l'incertitude, y entraîne ainsi ceux à qui l'histoire est adressée.

3 Suivant une démarche analytique rigoureuse, l'auteur présente des contre-exemples afin de tester la pertinence du modèle narratif qu'il vient de dégager sur la base de plusieurs récits. Deux cas sont exposés qui contreviennent aux règles observées: présence de rétrospections, profusion de détails non pertinents par rapport à l'énigme, absence de répétition, et silence sur l'imperception dans un cas, présence sonore dans l'autre. Or ces cas se sont avérés être des mystifications, confirmant la robustesse du schéma narratif proposé. Dans celui de Cergy-Pontoise, dont l'un des trois protagonistes aurait été enlevé par des extraterrestres la nuit du 26 novembre 1979, « la pente littéraire » du récit s'est développée sous la plume d'un auteur de sciencefiction, Jimmy Guieu, donnant lieu à la rumeur d'une destruction imminente de la Terre. Pourraient cependant y réchapper ceux qui se rendraient le 15 août 1980 sur les lieux de «l'enlèvement » afin d'entrer en contact avec des extraterrestres capables de les sauver du cataclysme. Plus de 500 personnes se rassemblèrent le jour dit au lieu dit, confirmant que « ces êtres de langage » (p. 106) n'en sont pas moins susceptibles d'agir sur la réalité. Il ne faudrait pas pour autant en conclure qu'ils existent au-delà du langage, dans un monde qui serait différent du nôtre, comme pourrait nous y engager un certain tournant ontologique des sciences sociales.

4 Le développement littéraire de ce cas, présenté comme le document d'une expérience vécue, conduit l'auteur à préciser les relations entre récits directs et littérature. Les romans de science-fiction, le premier genre littéraire auquel on pense, sont centrés sur les extraterrestres, leurs intentions et leurs actions éventuelles, alors que ceux-ci n'apparaissent presque pas dans les récits directs. Récits dans lesquels on ne retrouve pas non plus les idées d'évolution et d'adaptation qui hantent l'imaginaire de la science-fiction. L'incertitude que le narrateur partage avec ceux auxquels il s'adresse, qui conclut invariablement les récits directs, rapproche en revanche ceux-ci du genre fantastique. Dans le fantastique comme dans les récits directs, la vue est le sens qui est sollicité en premier lieu. C'est la soudaine soustraction à la vue de la chose énigmatique qui empêche le témoin de la reconnaître et de l'identifier, suscitant des interprétations sur son origine non-humaine. Le fantastique caractériserait aussi certains récits de rencontres avec des fantômes et des esprits en Amazonie et en Mongolie, avec cette différence cependant que le recours à l'origine non-humaine de phénomènes étranges fait partie du sens commun dans les exemples ethnographiques non-européens tandis que l'institution qui recueille les récits directs d'événements extraterrestres l'exclut d'emblée.

5 Dans la seconde partie du livre, on voit justement comment l'incertitude à laquelle sont suspendus ces récits est prise en charge par des institutions spécialisées qui mènent des enquêtes afin de « remettre de l'ordre » : l'ordre de la paix pour ce qui est de l'enquête militaire, celui de l'identification nominale des responsables dans l'enquête policière 
des gendarmes et, enfin, l'ordre du savoir et de l'identification catégorielle du phénomène propre à l'enquête scientifique du Geipan. La plupart du temps, la résolution de l'énigme relève clairement de l'humain et se réduit à un concours de circonstances ayant brouillé la perception d'un objet ordinaire tels un cerf-volant, un feu ou un laser. Il en va de même pour certains événements atmosphériques ou météorologiques. Certains récits demeurent cependant inexpliqués, les enquêteurs butant contre ce que l'auteur propose de voir comme l'intrusion du fantastique dans la réalité : "Pourquoi ne pas accepter de vivre dans un monde où le récit fantastique aurait sa place, au-delà de la littérature, dans un système commun à tous, où l'on admettrait que tout ne soit pas tristement familier, et où l'on assumerait l'incertitude poétique de ce que nous vivons?» (p. 206). En somme, dirions-nous, pourquoi ne pourrait-on pas ré-enchanter le monde ? Dans la conclusion, Arnaud Esquerre propose de considérer les récits directs d'événements extraterrestres comme une variation structurale des récits de fantômes qui les auraient historiquement précédés.

L'ouvrage frappe par sa puissance de démonstration et il faut lire la note méthodologique pour prendre toute la mesure du projet qui nous est proposé. L'auteur y explique son choix d'une approche structurale après avoir éliminé les alternatives méthodologiques par le contexte (la propagation d'une rumeur par exemple), la culture (les extraterrestres comme des variantes locales et contemporaines d'autres invisibles universels), la croyance (comme mécanisme cognitif oscillant entre doute et adhésion) et enfin la pragmatique (focalisée sur les effets de l'énonciation). On comprend alors que Théorie des événements extraterrestres est conçu comme le second tome d'un diptyque, le premier, Prédire, étant consacré à la pratique de l'astrologie. On remarquera que les deux ouvrages entretiennent une relation d'opposition structurale, d'abord par leur méthodologie : le premier recourt à la pragmatique et montre la façon dont le savoir issu d'une consultation est une coproduction entre l'astrologue et le client, tandis que le second lui oppose une approche structurale et insiste au contraire sur l'absence d'un tel processus interactif - sans doute ce point pourrait-il être débattu par les ethnographes des rencontres avec des agents non-humains. Ils diffèrent ensuite par leur objet : tandis que l'astrologie vise à maîtriser et réduire l'incertitude face à l'avenir, le récit d'expériences extraterrestres réintroduit l'incertitude dans le quotidien et manifeste au contraire la tentation d'ouvrir une brèche dans un monde désenchanté. Dans l'un comme dans l'autre, c'est au fond le rapport des humains à la réalité et au langage que l'auteur explore.

\section{AUTEURS}

\section{ANNE DE SALES}

desales.anne@wanadoo.fr 